\title{
A COUNTEREXAMPLE TO A CONJECTURE ON AN INTEGRAL CONDITION FOR DETERMINING PEAK POINTS (COUNTEREXAMPLE CONCERNING PEAK POINTS)
}

\author{
Alfred P. HALlstrom
}

Let $X$ be a compact plane set. Denote by $R(X)$ the uniform algebra generated by the rational functions with poles off $X$ and by $H(X)$ the space of functions harmonic in a neighborhood of $X$ endowed with the sup norm. A point $p \in \partial X$ is a peak point for $R(X)$ if there exists a function $f \in R(X)$ such that $f(p)=1$ and $|f(x)|<1$ if $x \neq p$. Moreover, $p$ is a peak point for $H(X)$ (consider $\operatorname{Re} f$ ) and hence, by a theorem of Keldysh, $p$ is a regular point for the Dirichlet problem. Conditions which determine whether or not a point is a peak point for $R(X)$ are thus of interest in harmonic analysis. Melnikov has given a necessary and sufficient condition that $p$ be a peak point for $R(X)$ in terms of analytic capacity, $\gamma$; namely $p$ is a peak point for $R(X)$ if and only if

$$
\sum_{n=0}^{\infty} 2^{n} \gamma\left(A_{n p} \backslash X\right)=\infty . \quad A_{n p}=\left\{z: \frac{1}{2^{n+1}} \leqq|z-p| \leqq \frac{1}{2^{n}}\right\} .
$$

Analytic capacity is generally difficult to compute, so it is desirable to obtain more computable types of conditions. Let $X^{c}=C \backslash X$ and

$$
I=\left\{t \in[0,1]: z \in X^{c} \text { and }|z|=t\right\} .
$$

In this note the following conjecture, which can be found in Zalcman's Springer Lecture Notes and which is true for certain sets $X$, is shown to be false in general:

Conjecture. If $\int_{I} t^{-1} d t=\infty$ then 0 is a peak point for $R(X)$. lemma:

Our counterexample uses Melnikov's theorem and the following

Lemma. Given $0<a<b$ and $\log b / a<2 \pi$ there exists $a$ set $K_{a b}$ such that $K_{a b} \subset\{z: a \leqq|z| \leqq b\}, \gamma\left(K_{a b}\right)=0$ and $\left\{t: z \in K_{a b}\right.$ and $\left.|z|=t\right\}=$ $[a, b]$.

The author is indebted to the referee for the following proof.

Garnett in [3] showed that the "Cantor corner square" set $K$ constructed by removing all but the four corner squares of length 1/4 from the unit square, then removing all but the sixteen corner squares of length $1 / 16$ from 


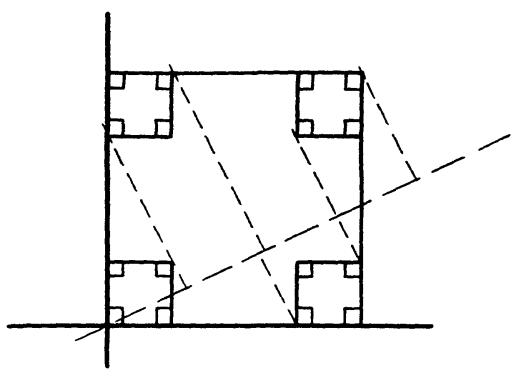

these four squares, etc., has zero analytic capacity while the projection on the line $y=x / 2$ is full, i.e., is the same as the projection of the unit square on that line. Thus given $0<a<b$ and $\log b / a<2 \pi$ there exists after a suitable rotation, expansion and translation a compact plane set $L_{a b}$ such that $\gamma\left(L_{a b}\right)=0$, the projection on the $x$-axis of $L_{a b}$ is $[\log a, \log b]$ and $L_{a b} \subset\{z: \log a \leqq x \leqq \log b, 0 \leqq y<2 \pi\}$. Let $K_{a b}=\left\{e^{z}: z \in L_{a b}\right\}$. Let $W$ be a small neighborhood of $L_{a b}$ such that the exponential map is $1-1$ on $W$ and $V=\left\{e^{z}: z \in w\right\}$. If $g$ is bounded and analytic on $V \backslash K_{a b}$ then $g\left(e^{z}\right)$ is bounded and analytic on $W \backslash L_{a b}$. Since $\gamma\left(L_{a b}\right)=0, g\left(e^{z}\right)$ extends analytically to $L_{a b}$ so $g$ extends analytically to $K_{a b}$. Thus $\gamma\left(K_{a b}\right)=0$. The other properties required of $K_{a b}$ obviously hold.

To construct our counterexample we choose open sets $U_{n} \subset A_{n 0}$ such that $K_{6 / 5 \cdot 1 / 2^{n+1,5 / 6} \cdot 1 / 2^{n}} \subset U_{n}$, and such that $\gamma\left(U_{n}\right)<1 / 4^{n}$. Let $X=\Delta(0,1) \backslash\left(\bigcup_{n=0}^{\infty} U_{n}\right)$. Then

$$
\sum_{n=0}^{\infty} 2^{n} \gamma\left(A_{n 0} \mid X\right)=\sum_{n=0}^{\infty} 2^{n} \gamma\left(U_{n}\right)<\infty
$$

so 0 is not a peak point for $R(X)$ by Melnikov's theorem. On the other hand,

$$
\int_{I} t^{-1} d t \geqq \sum_{n=0}^{\infty} \int_{6 / 5 \cdot 1 / 2^{n+1}}^{5 / 6 \cdot 1 / 2^{n}} t^{-1} d t=\sum_{n=0}^{\infty} \operatorname{Ln} \frac{\frac{5}{6} \cdot \frac{1}{2^{n}}}{\frac{6}{5} \cdot \frac{1}{2^{n+1}}}=\sum_{n=0}^{\infty} \operatorname{Ln} \frac{50}{60}=\infty .
$$

If we choose $U_{n}$ such that $\gamma\left(U_{n}\right) \leqq 1 /\left(2^{n}\right)^{2 n}$ then 0 supports bounded point derivations of all orders for $R(X)$, see [4], so 0 is indeed far from being a peak point for $R(X)$. The question remains whether 0 might be a regular point for the Dirichlet problem. We note that this could only happen if the set of representing measures $M_{x}$ for $R(K)$ at $x \in X^{0}$ is not norm compact, see [1].

\section{REFERENCES}

1. S. Fisher, Norm compact sets of representing measures, Proc. Amer. Math. Soc., 19 (1968), 1063-1068. 
2. J. Garnett, Analytic capacity and measure, University of California, preprint p. 8795 .

3. — Positive length but zero analytic capacity, Proc. Amer. Math. Soc., 21 (1970), 696-699.

4. A. P. Hallstrom, On bounded point derivations and analytic capacity, J. Functional Analysis, 4 (1969).

5. M. V. Keldysh, On the solvability and stability of the Dirichlet problem, Uspehi Mat. Nauk., 8 (1941), 171-231.

6. M. S. Melnikov, Analytic capacity and the Cauchy integral, Soviet Math. Dokl., 8 (1967), 20-23.

7. L. Zalcman, Analytic capacity and rational approximation, Lecture Notes in Mathematics 50, Berlin, 1968, p. 130.

Received August 18, 1972. This work was partially supported by NSF Grant No. GP-616508.

UNIVERSITY OF WASHINGTON 
